\title{
Symbolic-Numeric Completion of Differential Systems by Homotopy Continuation
}

\author{
Greg Reid*
}

Jan Verschelde ${ }^{\dagger}$

\begin{abstract}
Two ideas are combined to construct a hybrid symbolicnumeric differential-elimination method for identifying and including missing constraints arising in differential systems. First we exploit the fact that a system once differentiated becomes linear in its highest derivatives. Then we apply diagonal homotopies to incrementally process new constraints, one at a time. The method is illustrated on several examples, combining symbolic differential elimination (using rifsimp) with numerical homotopy continuation (using $p h \boldsymbol{c}$ ).
\end{abstract}

Categories and Subject Descriptors: G.1.8

General Terms: Algorithms, Design

Keywords: Component of solutions, diagonal homotopy, DAE (Differential Algebraic Equation), differential elimination, hidden constraint, homotopy continuation, numerical algebraic geometry, numerical jet geometry, path following, polynomial system, witness set.

\begin{abstract}
*Ontario Research Centre for Computer Algebra, c/o Department of Computer Science, University of Western Ontario, London, Ontario, N6A 5B7, Canada. Email: reid@uwo.ca. URL: http://www.orcca.on.ca/ reid. Support from NSERC is gratefully acknowledged.

${ }^{\dagger}$ Department of Mathematics, Statistics, and Computer Science, University of Illinois at Chicago, 851 South Morgan (M/C 249), Chicago, IL 60607-7045, U.S.A. Email: jan@math.uic.edu or jan.verschelde@na-net.ornl.gov. URL: http://www.math.uic.edu/ ${ }^{\sim}$ jan. This material is based upon work supported by the National Science Foundation under Grant No. 0134611 and Grant No. 0410036.

${ }^{\ddagger}$ Centre for Experimental and Computational Mathematics, Simon Fraser University, Burnaby, British Columbia, Canada. Email: wittkopf@cecm.sfu.ca. URL: http://www.cecm.sfu.ca/ wittkopf. Support from NSERC is gratefully acknowledged.

$\S$ Ontario Research Centre for Computer Algebra, c/o Department of Applied Mathematics, University of Western Ontario, London, Ontario, N6A 5B7, Canada. Email: wwu25@uwo.ca. Support from NSERC is gratefully acknowledged.
\end{abstract}

Permission to make digital or hard copies of all or part of this work for personal or classroom use is granted without fee provided that copies are not made or distributed for profit or commercial advantage and that copies bear this notice and the full citation on the first page. To copy otherwise, to republish, to post on servers or to redistribute to lists, requires prior specific permission and/or a fee.

ISSAC'05, July 24-27, 2005, Beijing, China.

Copyright 2005 ACM 1-59593-095-705/0007 ...\$5.00.

\author{
Allan Wittkopf ${ }^{\ddagger} \quad$ Wenyuan $\mathrm{Wu}^{\S}$
}

\section{INTRODUCTION}

Over and under-determined systems of ODE and PDE arise in applications such as constrained multibody mechanics and control systems (e.g. differential-algebraic equations (DAE) arise in constrained Lagrangian mechanics [20]).

Much progress has been made in exact differential elimination methods, theory and algorithms for nonlinear systems of PDE. For example see Boulier et al. [3], Chen and Gao [5], Hubert [9], Mansfield [12], Seiler [20], Reid, Rust et al. [18], Wu [30]. Such methods enable the identification of all the hidden constraints for a system of PDE and the automatic statement of an existence and uniqueness theorem for its solutions. They give a geometrical view of its solution space $[17,20]$ and enable the determination of its symmetry properties. They enable the computation of initial data and associated formal power series solutions in the neighborhood of a point. Algorithmic membership tests (specifically in the radical of a differential ideal) can be given [3, 9]. They can ease the difficulty of numerical solution of DAE systems [26].

This paper is a sequel to [14] and [7] in which we develop theory and methods for using numerical homotopy continuation methods in the differential elimination process to identify missing constraints for systems of differential equations. In [14] such methods were first introduced by combining the Cartan-Kuranishni approach with homotopy methods. During the application of that approach all equations are differentiated up to the current highest derivative order, resulting in potentially large numbers of PDE. These PDE are treated as polynomial equations in jet space, and their large number implies that the number of continuation paths that must be tracked can be impractically large.

In this paper we process the leading linear PDE using the rifsimp algorithm [29] and the leading nonlinear PDE using PHCpack [27], applying diagonal homotopies [23]. The correctness of our results can be certified if the constraints are free from multiplicities and the numerical representations are well conditioned.

This paper is organized as follows. In $\S 2$ we introduce some basic material for symbolic differential elimination, and in $\S 3$ we give a short overview on recent developments in homotopy methods. In $\S 4$ we present our method and briefly outline some optimizations in $\S 5$. Examples are given in $\S 6$ and concluding remarks in $\S 7$.

\section{SYMBOLIC DIFFERENTIAL ELIMINATION}

Consider a polynomially nonlinear system of PDE $R=$ $\left(R^{1}, \ldots, R^{l}\right)=0$ with independent variables $x=\left(x_{1}, \ldots, x_{n}\right)$ 
and dependent variables $u=\left(u^{1}, \ldots, u^{m}\right)$ over $\mathbb{C}$ with coefficients from some computable extension of $\mathbb{Q}$. As in $[3$, $18,20]$ solutions and derivatives are replaced by formal (jet) variables, allowing manipulation of equations without first assuming that solutions exist [13]. In particular, denoting the $p$-th order jet variables corresponding to derivatives as $u$, the jet variety of a $q$ th order system in $J^{q}=\mathbb{C}^{n_{q}}$ is

$$
V(R):=\left\{(x, u, \underset{1}{u}, \ldots, \underset{q}{u}) \in J^{q}: R(x, u, \underset{1}{u}, \ldots, \underset{q}{u})=0\right\} .
$$

Here $n_{q}=n+m\left(\begin{array}{c}n+q \\ q\end{array}\right)$ is the number of independent variables, dependent variables and derivatives of order less than or equal to $q$. We restrict to the subset of the variables of $J^{q}$ that actually appear in the given system.

EXAMPLE 2.1. Throughout this article we use the following running example, first introduced in [16], see also [7]:

$\frac{\partial^{2} u(x, y)}{\partial y^{2}}-\frac{\partial^{2} u(x, y)}{\partial x \partial y}=0,\left(\frac{\partial u(x, y)}{\partial x}\right)^{r}+\frac{\partial u(x, y)}{\partial x}-u(x, y)=0$.

For the case $r=2$, this is a differential polynomial system $R=\left(u_{y y}-u_{x y}, u_{x}^{2}+u_{x}-u\right)=0$ in the jet space of second order $J^{2}=\mathbb{C}^{8}$ and has jet variety $V(R)=\left\{\left(x, y, u, u_{x}\right.\right.$, $\left.\left.u_{y}, u_{x x}, u_{x y}, u_{y y}\right) \in J^{2}: u_{y y}-u_{x y}=0, u_{x}^{2}+u_{x}-u=0\right\}$.

The rifsimp algorithm [18, 29] takes on input a ranking of partial derivatives. A ranking of derivatives [18] is a total ordering on the set of all jet variables (excluding independent variables) that is invariant under differentiation and satisfies a positivity condition.

Definition 2.2 (Highest Derivative). The highest derivative of a $\mathrm{PDE} \psi$ is the largest derivative appearing in the PDE in the ranking. A PDE is classified as leading linear or leading nonlinear with respect to a ranking according as whether it is linear or not in its highest derivative.

EXAMPLE 2.3. Consider the ranking of partial derivatives:

$$
u \prec u_{x} \prec u_{y} \prec u_{x x} \prec u_{x y} \prec \cdots
$$

Then (2) has $\operatorname{HD}\left(u_{y y}-u_{x y}\right)=u_{y y}$ and $\operatorname{HD}\left(u_{x}^{2}+u_{x}-u\right)=u_{x}$. Hence $u_{y y}-u_{x y}$ is leading linear and $u_{x}^{2}+u_{x}-u$ is leading nonlinear with respect to the ranking.

Definition 2.4 (Formal Total Derivative). The formal total derivative is $D_{x_{j}}=\frac{\partial}{\partial x_{j}}+\sum_{\ell=1}^{m} u_{x_{j}}^{\ell} \frac{\partial}{\partial u^{\ell}}+\cdots$ Given a list of equations $N=0, D(N)$ is the list of first order total derivatives of all equations of $N$ with respect to all independent variables (i.e. $D(N):=\left[D_{x_{j}} N_{k}\right]$ ) and forms a single prolongation of $N$.

For Example 2.1 with $N=u_{x}^{2}+u_{x}-u=0$ and formal total derivatives $D_{x}$ and $D_{y}$ we have:

$$
D(N)=\left[2 u_{x} u_{x x}+u_{x x}-u_{x}, 2 u_{x} u_{x y}+u_{x y}-u_{y}\right] .
$$

Implementations of symbolic differential elimination algorithms have devoted much attention to decreasing the order of such prolongations, while still determining all the obstructions to the existence of formal power series solutions (e.g. see $[20,29])$.

As input the rifsimp algorithm takes a polynomially nonlinear system of PDE, together with a ranking of derivatives [18]. It partitions the system into two disjoint sets:
PDE which are linear in their highest derivatives with respect to the ranking, and its complement the leading nonlinear PDE. During its execution these two sets are continually updated until they satisfy certain theoretical conditions for termination $[18,29]$. One condition is that the integrability conditions, after reduction with respect to the leading nonlinear PDE, should lie in the algebraic ideal generated by the leading nonlinear PDE. Also the once-differentiated set of the leading nonlinear $\mathrm{PDE}$, after reduction with respect to the leading linear PDE, should lie in that ideal. Violation of these conditions gives new equations which are appended to the system, and the process above is repeated. In existing implementations [29] the membership is symbolically tested using Gröbner Bases and Triangular Set methods.

\section{NUMERICAL ALGEBRAIC GEOMETRY}

Our tool to numerically solve polynomial systems is homotopy continuation. Homotopy methods define families of systems, embedding a system to be solved in a homotopy, connecting it to a start system whose solutions are known. Continuation methods are then applied to track the paths defined by the homotopy, leading to the solutions. By random choices of constants in the homotopy one can prove that, except for an algebraic set of bad choices of constants, singularities and diverging paths can only occur at the end of the paths, when the system to be solved has singular solutions or fewer solutions than the generic root count.

In [24], a new field "Numerical Algebraic Geometry" was described which led to the development of homotopies to describe all irreducible components (all meaning: for all dimensions) of the solution set of a polynomial system. We briefly list key ingredients of this developing field, see also [25]:

1) Witness Sets: are the key data in a numerical irreducible decomposition. A witness set for a $k$-dimensional solution component consists of $k$ random hyperplanes and all isolated solutions in the intersection of the component with those hyperplanes. The degree of the solution component equals the number of witness points. Witness sets are equivalent to lifting fibers in a geometric resolution [10].

2) Cascade of Homotopies: Candidate witness points are computed efficiently using a cascade of homotopies, peeling off the hyperplanes in going from high to lower dimensional solution components. This idea of cutting with hyperplanes to determine the dimensions of solution components appeared in Giusti and Heintz [6].

3) Factorization. Using monodromy loops, certified by linear traces, a pure dimensional solution set is factored into irreducibles. See e.g. [4] for the related approximate multivariate polynomial factorization.

4) Diagonal Homotopies. We intersect two solution components: $A$ and $B$. Let $A$ be defined by polynomial equations $f(u)=0$, cut by hyperplanes $K(u)=0$, and $B$ similarly defined by $g(v)=0$ and $L(v)=0$. Consider the homotopy

$$
H(u, v, t)=\left[\begin{array}{c}
f(u)=0 \\
g(v)=0 \\
K(u)=0 \\
L(v)=0
\end{array}\right](1-t)+\left[\begin{array}{c}
f(u)=0 \\
g(v)=0 \\
u-v=0 \\
M(u)=0
\end{array}\right] t
$$

For $t=0$, we start at the product of the two witness sets $A \times B$. At $t=1$, we are at the diagonal 
$u-v=0$. The above homotopy is just a cartoon description of diagonal homotopies, see [23]. Intersecting components is done symbolically in the geometric resolution, see [10].

These methods have been implemented in PHCpack [27], see [22] for a description of some of its added capabilities. An interface to PHCpack within Maple is described in [11].

\section{SYMBOLIC-NUMERIC COMPLETION ALGORITHM}

Pseudo-code is given for the main algorithm HybridRif and its subroutines.

\subsection{Using Witness Sets}

A basic step of our method is to detect whether a new constraint lowers the dimension of some component of the current system. As we append constraints, the general membership test of [22] simplifies to substituting the witness points of the current constraints into the presumed new constraint.

Algorithm 4.1. $W^{\text {new }}=$ MembershipTest $(W, p, \epsilon)$

Input: $\quad W i t n e s s$ set $W$; a polynomial $p$; a tolerance $\epsilon$.

Output: $W^{n e w}=\{w \in W:|p(w)|>\epsilon\}$.

For correctness of our methods we need to test whether the constraints are free from singularities. Numerically this is done by setting thresholds on the condition numbers of the witness points. While ill-conditioned points are not necessarily points with with multiplicity $>1$ (extreme values of coefficients can lead to a bad scaling), for our current homotopy methods there is no difference in practice.

\section{Algorithm 4.2. boolean $=$ IsReduced $(W, \epsilon)$ \\ Input: Witness set $W$; $\epsilon$ tolerance on inverse condition number. \\ Output: true if all points in $W$ have good condition; false otherwise.}

A witness set for a hypersurface (defined by one multivariate polynomial) is obtained by cutting the hypersurface by a random line and applying a univariate root finder.

\section{Algorithm 4.3. $W=\boldsymbol{W i t n e s s S e t}(p, \epsilon)$ A polynomial $p$; a tolerance $\epsilon$. \\ Output: Witness set $W$ for $p$.}

Diagonal homotopies [23] are used to compute a numerical representation of the intersection of two solution components given by witness sets.

Algorithm 4.4. $W=$ Intersect $\left(W_{1}, W_{2}\right)$

Input: $\quad$ Witness sets $W_{1}, W_{2}$ representing $A, B$ resp.

Output: Witness $W$ representing $A \cap B$.

\subsection{Specification of rifsimp \& riflin}

In brief the rifsimp algorithm described in [18, 29] has the following input and output characteristics:

$$
\text { Algorithm 4.5. }[N, L, P]=\boldsymbol{r i f s i m p}(S, \prec)
$$

Input: $\quad S$, a system of polynomially nonlinear PDE and inequations over $\mathbb{Q}$, and a ranking $\prec$.

Output: $\quad[N, L, P]$, where
- L is a set of leading linear PDE in solved form with respect to its highest derivatives in the ranking $\prec$, where $\operatorname{HD}(L)$ are all distinct, and no member of $\operatorname{HD}(L)$ is a derivative of any other member;

- $N$ is a set of leading nonlinear PDE with no dependence on $\operatorname{HD}(L)$ or derivatives of $\operatorname{HD}(L)$;

- $P$ is a set of inequations (the pivots) with no dependence on on $\operatorname{HD}(L)$ or derivatives of $\operatorname{HD}(L)$;

- the integrability conditions of the leading linear PDE after reduction wrt $L$ are in $\langle N\rangle$;

- $D(N)$ after reduction wrt $L$ is in $\langle N\rangle$.

In addition, an existence and uniqueness theorem is provided for its output [18]. We work with a modified version of the rifsimp algorithm, called riflin, which works with only the leading linear part so no membership tests are performed and $D(N)$ is not computed. In contrast to rifsimp only a subset of all constraints are determined and an existence uniqueness result can not be stated.

\subsection{The main algorithm}

The symbolic algebraic equation manipulation in rifsimp is replaced with the numerical diagonal homotopy method.

$$
\text { Algorithm 4.6. }[N, P, L, \text { fail }]=\operatorname{HybridRif}(S, \prec, \epsilon)
$$

Input : $S$, a polynomially nonlinear system of differential equations and inequations over $\mathbb{Q}$ and a ranking $\prec$; a tolerance $\epsilon$.

Output: $[N, P, L$, fail $]$, where

$N$ : leading nonlinear part;

$P$ : pivots (inequations);

L: leading linear part;

fail: true if witness sets are not reduced.

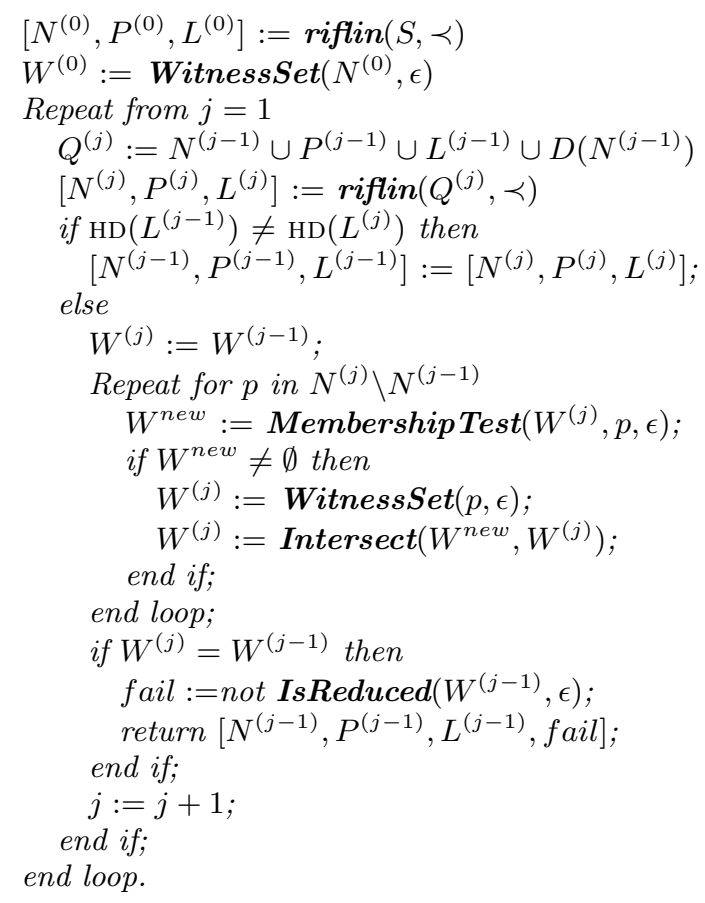

In practice $\boldsymbol{H y b r i d R i f}$ will abort reporting failure as soon as a witness set shows intolerably high condition numbers. 


\subsection{Termination Conditions}

Algorithm IsReduced is implemented using estimates for the inverse condition number of the Jacobian matrix at the witness points.

A standard Noetherian argument, which is a minor variation of that in [18], shows that the linear part $L^{(j)}$ must eventually stabilize. Further it is easily shown that

$$
\operatorname{HD}\left(L^{(j)}\right)=\operatorname{HD}\left(L^{(j-1)}\right) \Rightarrow P^{(j)}=P^{(j-1)} .
$$

The condition MembershipTest $\left(W^{(j-1)}, N^{(j)}, \epsilon\right)=\emptyset$ used to terminate HybridRif corresponds to the symbolic test involving the difference of two varieties:

$$
V\left(N^{(j)}\right) \backslash V\left(P^{(j-1)}\right) \supseteq V\left(N^{(j-1)}\right) \backslash V\left(P^{(j-1)}\right) .
$$

As HybridRif will fail when it encounters singularities or ill-conditioned representations, its termination is not absolute as is the case when the symbolic conditions (5) and (6) are applied. However, when HybridRif terminates without failure, the final witness set can be certified as follows: every witness point is an approximate zero in the sense of [2].

\section{OPTIMIZATIONS}

It will be advantageous, but not theoretically necessary, to remove redundant equations. A polynomial is redundant if after its removal the geometry of the solution set has not changed. By repeated calls to Algorithm 4.1, we can implement the following.

$$
\begin{array}{cl}
\text { Algorithm } 5.1 . & N^{\text {new }}=\operatorname{Shrink}(N, W, \epsilon) \\
\text { Input: } & N \text { a set of polynomials; } \\
& W \text { witness set representing } N^{-1}(0) ; \\
& \epsilon \text { is tolerance for Algorithm 4.1. }
\end{array}
$$

Output: $N^{\text {new }}$ cuts out same solution set as $N$.

The number of paths followed by homotopy methods is perhaps the most important aspect of their computational cost. In the case of dense polynomial square systems, this number is given by the Bézout degree. As a system is prolonged (differentiated) the number of equations can grow dramatically and the product of the degrees of these equations (their Bézout degree) can grow even more explosively. Thus methods for decreasing this number are a priority in the development of homotopy methods for PDE systems.

It is also advantageous to fix the value of the independent variables to random numbers: $x=\hat{x}$ where $x$ belongs to the space of independent variables $X$. Extended graphs of solutions of PDE belong to components which are fibred over $X$. This condition is ensured for each component of $V(N) \subseteq$ $J^{q}$ that is fibred over $X$. Let $\pi_{X}$ denote the projection onto $X$, that is $\pi_{X}(x, v)=x$ where $v=(u, \ldots, \underset{q}{u})$. Thus at neighborhoods $\mathcal{O}(\hat{x}, \hat{v})$ of regular points $(\hat{x}, \hat{v}) \in V(N)$ we have $\operatorname{dim}\left(\pi_{X} \mathcal{O}(\hat{x}, \hat{v}) \cap V(N)\right)=\operatorname{dim} X$. Equivalently we have $\operatorname{dim} \pi_{X} T_{(\hat{x}, \hat{v})} V(N)=\operatorname{dim} X=n$ where $T_{(\hat{x}, \hat{v})} V(N)$ is the tangent space to $V(N)$ at $(\hat{x}, \hat{v})$.

Suppose we are given a system with variety $C$ and a hypersurface $S$. We can already test $S \supseteq C$, by use of the algorithm MembershipTest and substitution of generic points, but the number of continuation paths can be impractically large. Instead we set $x=\hat{x}, C_{\hat{x}}=\{(x, v) \in C: x=\hat{x}\}=$ $\ell_{\hat{x}} \cap C$ and $S_{\hat{x}}=\{(x, v) \in S: x=\hat{x}\}=\ell_{\hat{x}} \cap S$ where $\ell_{\hat{x}}=\{(\hat{x}, v)\}$ is a linear space. By application of MembershipTest and substitution of generic points we can determine if $S_{\hat{x}} \supseteq C_{\hat{x}}$ by following far fewer continuation paths.
In general however this does not necessarily imply $S \supseteq C$. For example consider $S=\{(x, u):(x-3)(u-1)=0\}$ and $C=\{(x, u):(u-2)=0\}$ then with $\hat{x}=3, S_{\hat{x}} \supseteq C_{\hat{x}}$, but $S \nsupseteq C$. But note that components of form such as $x-3=0$ are not fibred over $X$ and are not of interest for PDE, since they imply that the 'independent variables' are instead dependent on each other. Such non-fibred components are avoided, with high probability, by setting $x=\hat{x}$.

Assume $C$ has only one component fibred over $X$ and $S_{\hat{x}} \supseteq C_{\hat{x}}$. If $S \nsupseteq C$, then $C \cap S$ is a proper algebraic subset of $C[21]$ which means $\operatorname{dim} C>\operatorname{dim}(C \cap S)$ and since $C$ is fibred over $X, C_{\hat{x}}$ is not empty. Therefore $\operatorname{dim} C_{\hat{x}}>$ $\operatorname{dim}\left(C \cap S \cap \ell_{\hat{x}}\right)=\operatorname{dim}\left(C_{\hat{x}} \cap S_{\hat{x}}\right)$, contradicting $S_{\hat{x}} \supseteq C_{\hat{x}}$, so $S$ must contain the whole component of $C$. This technique can often dramatically decrease the Bézout bound of the system and number of paths for the witness set by homotopies in MembershipTest. Note that the degree $d$ of a $\mathrm{PDE}$, when the independent variables are fixed to constants, is invariant under prolongation. Hence the Bézout degree of the prolongation of a single PDE, is $d^{N}$ where $N$ is the number of PDE in the prolongation.

\section{EXAMPLES}

\subsection{Illustrative Example}

The simple illustrative system (2) with $r=2$ has

$$
S:=\left[u_{y y}-u_{x y}=0, u_{x}^{2}+u_{x}-u=0\right]
$$

on entry into HybridRif. We assume the ranking is given by (3). Since the independent variables $x, y$ do not appear explicitly they are not used in dimension counts.

At the first iteration, applying riflin to $S$ yields the single leading linear PDE in the solved form $L^{(0)}$, and a single leading nonlinear PDE $N^{(0)}$ :

$$
\begin{array}{lll}
N^{(0)} & P^{(0)} & L^{(0)} \\
u_{x}^{2}+u_{x}-u=0 & \emptyset & u_{y y}=u_{x y}
\end{array}
$$

We first calculate $D\left(N^{(0)}\right)=\left[2 u_{x} u_{x x}+u_{x x}-u_{x}, 2 u_{x} u_{x y}+\right.$ $\left.u_{x y}-u_{y}\right]$ then

$$
Q^{(1)}=L^{(0)} \cup P^{(0)} \cup N^{(0)} \cup D\left(N^{(0)}\right)
$$

and apply riflin to $Q^{(1)}$ to obtain

$$
\begin{array}{lll}
N^{(1)} & P^{(1)} & L^{(1)} \\
u_{x}^{2}+u_{x}-u=0 & \left(2 u_{x}+1\right) \neq 0 & u_{y y}=\frac{u_{y}}{2 u_{x}+1} \\
u_{y}^{2}-u_{y} u_{x}=0 & & u_{x y}=\frac{u_{y}}{2 u_{x}+1} \\
u_{y} u_{x}-u_{y}^{2}=0 & & u_{x x}=\frac{u_{x}}{2 u_{x}+1}
\end{array}
$$

We remove the obvious duplicate equation in $N^{(1)}$ by a simple implementation of Algorithm 5.1 although this is not necessary for the correctness and termination of HybridRif. Next we check whether the leading linear part is stable or not. Since $\operatorname{HD}\left(L^{(1)}\right)=\left[u_{y y}, u_{x y}, u_{x x}\right] \neq \operatorname{HD}\left(L^{(0)}\right)=\left[u_{y y}\right]$ we return to the beginning of the major loop. We first compute

$$
\begin{array}{r}
D\left(N^{(1)}\right)=\left[2 u_{x} u_{x x}+u_{x x}-u_{x}, 2 u_{x} u_{x y}+u_{x y}-u_{y},\right. \\
-u_{x y}\left(u_{x}-u_{y}\right)-u_{y}\left(u_{x x}-u_{x y}\right), \\
\left.-u_{y y}\left(u_{x}-u_{y}\right)-u_{y}\left(u_{x y}-u_{y y}\right)\right]
\end{array}
$$

then $Q^{(2)}=L^{(1)} \cup P^{(1)} \cup N^{(1)} \cup D\left(N^{(1)}\right)$. Next riflin is applied to $Q^{(2)}$ which after removing redundant equations 
gives:

$$
\begin{array}{lll}
N^{(2)} & P^{(2)} & L^{(2)} \\
u_{x}^{2}+u_{x}-u=0 & \left(2 u_{x}+1\right) \neq 0 & u_{y y}=\frac{u_{y}}{2 u_{x}+1} \\
u_{y} u_{x}-u_{y}^{2}=0 & & u_{x y}=\frac{u_{y}}{2 u_{x}+1} \\
& & u_{x x}=\frac{u_{x}}{2 u_{x}+1}
\end{array}
$$

Here $\operatorname{HD}\left(L^{(2)}\right)=\operatorname{HD}\left(L^{(1)}\right)$, so the membership test is applied to $N^{(2)}$ to test $V\left(N^{(2)}\right) \backslash V\left(P^{(2)}\right) \supseteq V\left(N^{(1)}\right) \backslash V\left(P^{(1)}\right)$.

First we compute the witness set of each polynomial in $N^{(1)}$ in $\left(u, u_{x}, u_{y}\right)$-space by WitnessSet. There are two paths to be followed for each polynomial. Then the witness set $W^{(1)}$ for $N^{(1)}$ is computed by Intersect, yielding four witness points resulting from tracking 4 paths. During the application of MembershipTest points in $W^{(1)}$ are evaluated in the system $N^{(2)}$. Since IsReduced $\left(W^{(1)}, \epsilon\right)=$ true and MembershipTest $\left(W^{(1)}, N^{(2)}, \epsilon\right)=\emptyset$, the termination conditions are met and the algorithm returns $\left[N^{(1)}, P^{(1)}, L^{(1)}\right]$.

For this example it can be checked that the outputs of HybridRif and the fully symbolic algorithm rifsimp are the same. In $\S 6.2$ an example is given where the outputs of HybridRif and rifsimp differ.

Comparison with a Numerical Geometrical Completion Method: Here we compare HybridRif with a numerical geometrical completion method $[1,7,14]$ which is a variation of the symbolic Cartan-Kuranishi method [13, 20]. In [7] the first application of the interpolation-free method of [14] is given to the example system above. The method when applied to an input system $R$ involves computing $\operatorname{dim} \pi^{\ell} D^{k} R$ where $\pi: J^{q} \rightarrow J^{q-1}$ is the usual projection until the criteria of projected involution [1] are satisfied. The output of the method of [7] consists of

$$
\begin{aligned}
& \phi^{1}=0, \phi^{2}=0, \\
& D_{x}\left(\phi^{1}\right)=0, D_{y}\left(\phi^{1}\right)=0, D_{x}\left(\phi^{2}\right)=0, D_{y}\left(\phi^{2}\right)=0, \\
& D_{x x}\left(\phi^{2}\right)=0, D_{x y}\left(\phi^{2}\right)=0, D_{y y}\left(\phi^{2}\right)=0
\end{aligned}
$$

where $R=\left[\phi^{1}=u_{y y}-u_{x y}=0, \phi^{2}=u_{x}^{2}+u_{x}-u=0\right]$ is the input system above. In [7] the following dimensions are computed using homotopy continuation:

$$
\begin{aligned}
& \operatorname{dim}(R)=2 \quad \operatorname{dim}(D R)=1 \quad \operatorname{dim}\left(D^{2} R\right)=1 \\
& \operatorname{dim} \pi(D R)=1 \quad \operatorname{dim} \pi\left(D^{2} R\right)=1 \\
& \operatorname{dim} \pi^{2}\left(D^{2} R\right)=1
\end{aligned}
$$

and show $\pi(D R)$ is an involutive system. In the computations, the worst Bézout number that appears is 64 which is much bigger than 4 , the number of continuation paths that had to be followed in the application of HybridRif above.

\subsection{System for Discrete Symmetries$$
\text { of } \mathbf{u}_{\mathrm{xx}}=\frac{1}{\mathrm{x}} \mathbf{u}_{\mathrm{x}}+\frac{4}{\mathrm{x}^{3}} \mathbf{u}^{2}
$$

Reference [15] solves the problem of determining the full diffeomorphism pseudogroup of point transformations $(x, u) \mapsto$ $(\hat{x}, \hat{u})$ of the form $\hat{x}=X(x, u), \hat{u}=U(x, u)$, leaving invariant the ODE $u_{x x}=\frac{1}{x} u_{x}+\frac{4}{x^{3}} u^{2}$. Requiring that these transformations leave the ODE invariant leads [15] to a system of nonlinear PDE for the unknown functions $X, U$ :

$$
\begin{aligned}
& 4 U^{2} X_{u}^{3}-X^{3} X_{u} U_{u u}+X^{3} U_{u} X_{u u}+X^{2} U_{u} X_{u}^{2}=0 \\
& X^{2} U_{x} X_{u}^{2}+2 X^{3} U_{u} X_{x u}-X^{3} X_{x} U_{u u}-2 X^{3} X_{u} U_{x u}+2 X^{2} U_{u} X_{x} X_{u} \\
& \quad+12 U^{2} X_{x} X_{u}^{2}+X^{3} U_{x} X_{u u}=0 \\
& x^{3} X^{3} U_{x} X_{x x}-4 u^{2} X^{3} U_{u} X_{x}-x^{3} X^{3} U_{x x} X_{x}+x^{3} X^{2} U_{x} X_{x}^{2} \\
& \quad+4 u^{2} X^{3} U_{x} X_{u}+4 x^{3} U^{2} X_{x}^{3}=0 \\
& \quad 2 x X^{3} U_{x} X_{x u}+X^{3} U_{x} X_{u}-x X^{3} U_{x x} X_{u}+2 x X^{2} U_{x} X_{x} X_{u}-X^{3} U_{u} X_{x} \\
& \quad+x X^{2} U_{u} X_{x}^{2}-2 x X^{3} U_{x u} X_{x}+x X^{3} U_{u} X_{x x}+12 x U^{2} X_{x}^{2} X_{u}=0
\end{aligned}
$$

augmented with the condition that the Jacobian of the transformation does not vanish: $X_{x} U_{u}-X_{u} U_{x} \neq 0$.

Application of the HybridRif Algorithm with the ranking graded first by total order of derivative, then with $\partial_{u} \prec$ $\partial_{x}$ and finally lexicographically with $U \prec X$, i.e.:

$$
U \prec X \prec U_{u} \prec X_{u} \prec U_{x} \prec X_{x} \prec \cdots
$$

gives the leading linear system

$$
\begin{aligned}
X_{x x}= & \frac{6 X^{3}-5 x^{2} X_{x} U_{u} X-x^{3} U_{u} X_{x}^{2}}{5 x^{3} X U_{u}}, \quad X_{u}=0 \\
U_{x x}= & \left(20 x^{3} U^{2} U_{u} X_{x}^{3}-20 u^{2} X^{3} U_{u}{ }^{2} X_{x}-5 x^{2} U_{u} X^{3} U_{x} X_{x}\right. \\
& \left.+4 x^{3} U_{x} X^{2} U_{u} X_{x}{ }^{2}+6 U_{x} X^{5}\right) /\left(5 x^{3} U_{u} X_{x} X^{3}\right) \\
U_{x u}= & \frac{2 x^{3} U_{u} X_{x}^{2}-5 x^{2} X_{x} U_{u} X+3 X^{3}}{5 x^{3} X X_{x}} \\
U_{u u}= & 0
\end{aligned}
$$

together with the condition $U_{u} \neq 0, X_{x} \neq 0$. The constraint leading nonlinear equations found by HybridRif are:

$$
\begin{aligned}
& x^{3} U_{u} X_{x}{ }^{2}-X^{3}=0, \\
& -200 x^{3} u X_{x}{ }^{2} X^{3} U_{u}{ }^{2}+200 x^{6} X_{x}{ }^{4} U_{u}{ }^{2} U-27 x^{3} U_{u} X^{4} X_{x}{ }^{2}+36 X^{7} \\
& \quad-25 x^{4} U_{u}{ }^{2} X^{3} X_{x}{ }^{2}+16 x^{6} U_{u}{ }^{2} X X_{x}{ }^{4}=0, \\
& -200 x^{6} X_{x}{ }^{3} U_{u}{ }^{2} U_{x}-16 x^{6} U_{u}{ }^{2} X_{x}{ }^{4}+12 x^{3} U_{u} X^{3} X_{x}{ }^{2}+90 x X^{5} U_{u} \\
& \quad-1200 x^{3} X^{2} U_{u} X_{x}{ }^{2} U+680 x^{3} u X_{x}{ }^{2} X^{2} U_{u}{ }^{2}+85 x^{4} U_{u}{ }^{2} X^{2} X_{x}{ }^{2} \\
& \quad+720 u X^{5} U_{u}-171 X^{6}-200 x^{2} u X_{x} X^{3} U_{u}{ }^{2}=0, \\
& 432 u X^{10}+792 x^{4} X^{7} X_{x}{ }^{2} U_{u}+204 x^{7} X^{4} X_{x}{ }^{4} U_{u}{ }^{2}-60 x^{6} X^{5} X_{x}{ }^{4} U_{u} \\
& \quad-3600 x^{3} X^{7} X_{x}{ }^{2} U-200 x^{9} X^{2} X_{x}{ }^{5} U_{u}{ }^{2} U_{x}+200 x^{4} u X^{6} X_{x}{ }^{2} U_{u}{ }^{2} \\
& \quad-1200 x^{2} u X^{8} X_{x} U_{u}-2400 x^{6} X^{5} X_{x}{ }^{3} U_{x} U_{u}-2400 x^{6} X^{4} X_{x}{ }^{4} U_{u} U \\
& \quad+1632 x^{6} u X^{4} X_{x}{ }^{4} U_{u}{ }^{2}+6336 x^{3} u X^{7} X_{x}{ }^{2} U_{u}-800 x^{9} X_{x}{ }^{6} U_{u}{ }^{2} U^{2} \\
& \quad-1400 x^{5} u X^{5} X_{x}{ }^{3} U_{u}{ }^{2}+800 x^{6} u^{2} X_{x}{ }^{4} U_{u}{ }^{3} X^{3}-990 x^{3} X^{8} X_{x}{ }^{2} \\
& \quad+54 x X^{10}=0
\end{aligned}
$$

Application of the initial data algorithm [29] to the leading linear equations (17) above yields the following initial data

$$
\begin{aligned}
& X\left(x_{0}, u_{0}\right)=X^{0}, U\left(x_{0}, u_{0}\right)=U^{0}, \\
& X_{x}\left(x_{0}, u_{0}\right)=X_{x}^{0}, U_{x}\left(x_{0}, u_{0}\right)=U_{x}^{0}, U_{u}\left(x_{0}, u_{0}\right)=U_{u}^{0} .
\end{aligned}
$$

Then the existence and uniqueness theorem [18] implies that formal power solutions to the system exist at points where the constants $X^{0}, U^{0}, X_{x}^{0}, U_{x}^{0}, U_{u}^{0}$ satisfy the constraint nonlinear equations (18).

In this example $N^{(1)}$ consists of the first 3 nonlinear equations in (18) with degrees 6,13,12 respectively, and the corresponding linear part $\left(L^{(1)}\right)$ becomes stable. Next the witness set is constructed for $N^{(1)}$. Next $N^{(2)}$ is obtained with all 4 nonlinear equations of (18) with degrees 6, 13, 12 and 19 respectively. Application of MembershipTest shows that 
the fourth equation is geometrically new so the witness set of its intersection is computed using Intersect.

Because of the high total degree in this example, we use techniques to decrease the number the continuation paths followed by phc. The first technique is to specialize the independent variables to random fixed values as discussed in $\S 5$. In particular the degrees of the uncovered constraints (18) decrease dramatically from 6, 13, 12, 19 to 3, 7, 6, 10. A second key to success, was to use mixed volumes instead of Bézout Bounds. In particular in the application of diagonal homotopies, this decreased the number of paths needing to be followed for $N^{(1)}$ from $126(=3 \cdot 7 \cdot 6)$ to 3 and the number of paths for $N^{(2)}$ from $1260(=3 \cdot 7 \cdot 6 \cdot 10)$ to 4 .

Application of diagonal homotopies showed the existence of 1 dimensional components for the constraint nonlinear system ( 3 dimensional if we include $x, u$ in the dimension count). This agrees with the explicit computations in [15]. Denote by $\mathcal{G}_{\text {lie }}$ the Lie subgroup of symmetries in a connected component of the identity of the full symmetry group $\mathcal{G}$ of the ODE. Our dimensional computation correctly reveals the dimension of $\mathcal{G}_{\text {lie }}$ as 1 as determined by a more conventional linearized calculation in [15]. The degree determined by our calculations is 4 and corresponds to the cardinality of $\mathcal{G} / \mathcal{G}_{\text {lie }}$ which is in agreement with [15] (indeed there it is shown that the factor group is isomorphic to $\mathbb{Z}_{4}$ ). Further calculations using phc on the full constraint nonlinear system reveals that there are 4 degree one, one dimensional components (fixing $x, u$ to constants) whose equations can be interpolated if desired. These computations are again in agreement with the explicit ones in [15].

Interestingly high degree singular components of natural geometric origin violating the invertibility condition $X_{x} U_{u}-$ $X_{u} U_{x} \neq 0$ arose in our calculations and initially caused some numerical difficulties. Such components were excluded by inclusion of the invertibility condition. For the system above this is equivalent to $X_{x} U_{u} \neq 0$, since $X_{u}=0$. Consequently we also have $X \neq 0$ and $U \neq 0$.

Comparison with a Numerical Geometrical Completion Method: We compare HybridRif with a numerical geometrical completion method $[1,7,14]$ which is a variation of the symbolic Cartan-Kuranishi method [13, 20]. The method when applied to an input system $R$ (18) involves computing $\operatorname{dim} \pi^{\ell} D^{k} R$ until the criteria of projected involution [1] are satisfied. The system $R$ has Bézout number 12288 which is reduced to 1875 after substituting random values for the independent variables. The prolongation of $D R$ which has 18 equations with Bézout number 50096498540544. After specializing the independent variables it reduces to 177978515625 which was still too high.

Comparison with the rifsimp symbolic algorithm: Application of rifsimp with the ranking (16) yielded the leading linear system:

$$
\begin{aligned}
U_{x, x}= & \left(-16 x^{2} u U^{2}-4 x^{3} U^{2}-128 x u^{2} U^{2}+\right. \\
& -384 u^{2} x^{2} U_{x} U-16 x^{4} U_{x} U-160 x^{3} u U_{x} U+ \\
& +4 x^{2} u X U+16 x u^{2} X U+128 u^{3} X U-3 x^{4} X U_{x}+ \\
& \left.-80 u^{2} x^{2} U_{x} X-32 x^{3} u U_{x} X\right)\left(x^{3}(4 u+x)^{2}(X+8 U)\right) \\
X_{x}= & \left(U^{2}(4 U+X)(8 u+x)\right) / \\
& \left(u \left(32 u U^{2}+4 x U^{2}+8 x^{2} U_{x} U+32 u x U_{x} U\right.\right. \\
& \left.\left.+8 u X U+x X U+4 x u X U_{x}+x^{2} U_{x} X\right)\right) \\
X_{u}= & 0 \\
U_{u}= & \frac{X+8 U}{8 u+x}
\end{aligned}
$$

\begin{tabular}{|lcccccccc|}
\hline$d$ & 2 & 3 & 4 & 5 & 6 & 7 & 8 & 9 \\
\hline RAM $\mathbb{Z}$ & 0.24 & 0.6 & 1.4 & 2.5 & 8.3 & 16.5 & 128.7 & INC \\
RAM: $\mathbb{C}$ & 3.40 & 6.8 & 11.2 & 20.7 & 62.2 & INC & INC & INC \\
\hline
\end{tabular}

Table 1: rifsimp memory consumption (MB) applied to a class of random polynomial ODE $R\left(u_{x}, u\right)=0$ with integer coefficients, and a class with complex rational coefficients. Here $d=\operatorname{degree}(R)$. RAM=inc indicates the memory of machine was exhausted.

\begin{tabular}{|lccccccc|}
\hline$d$ & 2 & 5 & 8 & 11 & 14 & 17 & 20 \\
\hline RAM: $\mathbb{Z}$ & 1.5 & 1.8 & 2.0 & 2.1 & 2.2 & 2.3 & 2.5 \\
RAM: $\mathbb{C}$ & 1.5 & 1.8 & 2.0 & 2.0 & 2.2 & 2.3 & 2.6 \\
\hline
\end{tabular}

Table 2: phc memory consumption (MB) applied to a class of random polynomial ODE $R\left(u_{x}, u\right)=0$ with integer coefficients, and a class with complex rational coefficients. Here $d=\operatorname{degree}(R)$.

with the leading nonlinear equations

$$
\begin{array}{r}
32 u^{3} X U-64 u^{2} x^{2} U_{x} U-32 x u^{2} U^{2}-8 u^{2} x^{2} U_{x} X \\
+8 x u^{2} X U-32 u^{2} x^{3} U_{x}^{2}-2 x^{3} u U_{x} X-16 x^{3} u U_{x} U \\
+x^{2} u X U-12 u x^{4} U_{x}^{2}-8 x^{2} u U^{2}-x^{3} U^{2}-x^{5} U_{x}^{2}=0, \\
(u X-x U)(x X+4 u X+4 x U)=0
\end{array}
$$

and the inequations $X+8 U \neq 0, X \neq 0, U \neq 0$.

Unlike the example of $\S 6.1$, this differs from the result obtained by HybridRif. This discrepancy is resolved by noting that both systems define the the same locus in jet space.

Finally we note that Hydon [8] gives an elegant and efficient method which exploits the knowledge of the Lie group $\mathcal{G}_{\text {lie }}$ to considerably ease computation of the full group $\mathcal{G}$.

\subsection{Random first order $\operatorname{ODE} R\left(\mathbf{u}_{\mathbf{x}}, \mathbf{u}\right)=\mathbf{0}$}

In this section we apply our symbolic-numeric approach to a class of random ODE $R\left(u_{x}, u\right)=0$ for a single dependent variable $u$. The efficiency of this approach is compared with that of using the symbolic rifsimp algorithm. Differentiation of $R\left(u_{x}, u\right)=0$ gives $R_{u_{x}} u_{x x}+R_{u} u_{x}=0$. The following cases are easily obtained:

$$
\begin{aligned}
& \text { Case 1: } u_{x x}=-\frac{R_{u} u_{x}}{R_{u_{x}}}, R_{u_{x}} \neq 0, R\left(u_{x}, u\right)=0 \\
& \text { Case 2: } S_{2}=\left\{R=0, R_{u}=0, R_{u_{x}}=0\right\}, u_{x} \neq 0 \\
& \text { Case 3: } S_{3}=\left\{R(0, u)=0, R_{u_{x}}(0, u)=0\right\}, u_{x}=0
\end{aligned}
$$

For random differential polynomials $R$, system $S_{2}$ in (22) consists of two random polynomials in one variable and system $S_{3}$ in (22) consists of three random polynomials in two variables. For random $R$ systems $S_{2}$ and $S_{3}$ will be inconsistent with high probability and Case 1 will be the only consistent case. A full analysis of all the singular cases for such ODE is given in the classic work of Hubert [9].

Two subclasses of random ODE with degrees $d$ from 1 to 20 were considered. One subclass had random coefficients consisting of integers between -99 and 99, and the other random subclass had random coefficients consisting of complex numbers of the form $(a+b i) /(\max \{|a|,|b|\}+1)$ where $a, b$ are random integers between -10 and 10 .

The computations were carried out using Maple 9, and phc (release 2.3 beta) on a $1.5 \mathrm{GHZ}$ Pentium M, with 512 MB of RAM, running under Windows XP. As shown in Table 1 the RAM was exhausted at relatively low degree $d=9$, 


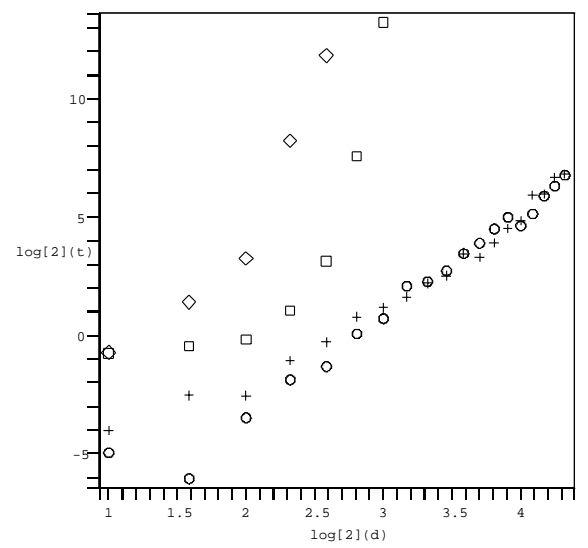

Figure 1: Time-Degree Statistics for a Random polynomial ODE $\mathbf{R}\left(\mathbf{u}_{\mathbf{x}}, \mathbf{u}\right)=\mathbf{0}$ plotted on a $\log -\log$ scale. $d=\operatorname{degree}(R)$ and $t$ is the time to apply the differential elimination process using: rifsimp (comp. coeffs. $\diamond$ ); rifsimp (integ. coeffs. $\square$ ); phc (comp. coeffs. + ); phc (integ. coeffs. o).

and this was dramatically worse for complex coefficients where exhaustion occurred at $d=7$. As seen in Table 2 RAM usage by $\boldsymbol{p h} \boldsymbol{c}$ was dramatically lower and more stable than that of rifsimp. While changing from random integer to complex coefficients barely affected the RAM consumed by $\boldsymbol{p h} \boldsymbol{c}$, it dramatically increased RAM usage by rifsimp.

Degree-time statistics for rifsimp and phc are shown in Figure 1. The positive concavity of the two curves for rifsimp indicates its complexity is more than polynomial. The approximately linear curves for $\boldsymbol{p h} \boldsymbol{c}$ in Figure 1 on the log$\log$ scale is typical for a polynomial-time method. However the worst case complexity of HybridRif is at least exponential, considering its application to systems of linear homogeneous PDE in a single dependent variable. In that case its output is isomorphic to a Gröbner Basis. Groundbreaking work on reducing the complexity to polynomial time for ODE was done by Sedglovacic [19]. The memory usage statistics show the discrepancy between $\boldsymbol{r i f s i m p}$ and $\boldsymbol{p h} \boldsymbol{c}$ growing with increasing degree, and when changing from integer to random complex coefficients. The symbolic differential elimination program Rosenfeld_Groebner had similar memory and time behavior to rifsimp on the random class of ODE.

\section{DISCUSSION}

Our method applies to intrinsic (exact) systems of polynomially nonlinear PDE and relies on splitting the system into a leading linear subsystem and its complement. Welldeveloped (linear) symbolic methods are applied to the leading linear part of the system. The success of this strategy enables the shrinking of the number of genuinely nonlinear equations that are dealt with by the numerical continuation methods. The use of diagonal homotopies allows handling the constraints incrementally, exploiting the structure of the leading nonlinear systems, and leads to a further decrease in the number of paths to be followed. Note that one could - at least in theory - replace the use of witness sets and diagonal homotopies in HybridRif by lifting fibers and using geometric resolutions [10]. In contrast to Gröbner methods, the fact that only geometrically new constraints are used means that generally fewer constraints need to be stored than would be required to represent the ideal. In addition, the maintenance of the constraints in their introduced form helps to preserve sparsity, and reduce equation and coefficient growth typical of Gröbner methods. It also allows flexibility in using alternative and sparse methods to control expression swell. Such methods include encoding the constraints by straight line programs, or using memory management based on ordered storage strategies [29] or directed acyclic graph structures as used by Lecerf in his implementation of the algorithms in [10].

The methods were applied to a number of examples starting with an easy illustrative example in $\S 6.1$. Secondly a system for discrete symmetries of moderate difficulty for symbolic methods was considered. Although the output was implicit, it illustrated that useful features of the symmetries could be extracted by the new hybrid methods (such as the number of discrete symmetries, and the degree of the components of the group). On this example, HybridRif was compared with a geometrical approach based on a numerical version of the Cartan-Kuranishi algorithm. It demonstrated that far fewer continuation paths were needed by HybridRif than the numerical geometrical method developed in earlier work.

Finally in $\S 6.3$ we considered a class of random first order ODE. On systems which are denser and of higher degree, numerical methods have an advantage while symbolic methods can perform better on lower degree, highly structured sparse systems. We caution that the sample size is too small to make emphatic statements. Certainly it indicates that there is scope to improve rifsimp's algebraic processing by using alternative symbolic and numeric algorithms.

This paper belongs to a series initiated in [28], continued in [14] and [7], aimed at developing "Numerical Jet Geometry", as a subfield of "Numerical Algebraic Geometry". Ultimately, this development will lead to methods enabling the practical processing of approximate input systems.

\section{REFERENCES}

[1] J. Bonasia, F. Lemaire, G. Reid, R. Scott, and L. Zhi. Determination of Approximate Symmetries of Differential Equations. Centre de Recherches Mathématiques, CRM Proceedings and Lecture Notes. Vol 39, pages 233-250, 2004.

[2] L. Blum, F. Cucker, M. Shub, and S. Smale. Complexity and Real Computation. Springer-Verlag, 1998.

[3] F. Boulier, D. Lazard, F. Ollivier, and M. Petitot. Representation for the radical of a finitely generated differential ideal. Proc. ISSAC 1995. ACM Press. 158-166, 1995.

[4] G. Chèze and A. Galligo. Four Lectures on Polynomial Absolute Factorization. In A. Dickenstein and I.Z. Emiris (Eds.), Solving Polynomial Equations: Foundations, Algorithms, and Applications. Volume 14 of Algorithms and Computation in Mathematics 14, Springer-Verlag, pages 339-392, 2005.

[5] Y. Chen and X.-S. Gao. Involutive Bases of Algebraic Partial Differential Equation Systems. Science in China (A), 33(2), page 97-113, 2003.

[6] M. Giusti and J. Heintz. La détermination de la dimension et des points isolées d'une variété 
algébrique peuvent s'effectuer en temps polynomial. In D. Eisenbud and L. Robbiano, eds., Computational Algebraic Geometry and Commutative Algebra, Cortona 1991, vol. XXXIV of Symposia Mathematica, pages 216-256. Camb. Univ. Press, 1993.

[7] K. Hazaveh, D.J. Jeffrey, G.J. Reid, S.M. Watt, and A.D. Wittkopf. An exploration of homotopy solving in Maple. Proc. of the Sixth Asian Symp. on Computer Math. (ASCM 2003). Lect. Notes Series on Computing by World Sci. Publ. 10 edited by Z. Li and W. Sit (Singapore/River Edge, USA) 145-162, 2003.

[8] P.E. Hydon. Discrete point symmetries of ordinary differential equations. $R$. Soc. Lond. Proc. Ser. A Math. Phys. Eng. Sci. 454 1961-1972, 1998.

[9] E. Hubert. Detecting degenerate cases in non-linear differential equations of first order. Theoretical Computer Science 187(1-2): 7-25, 1997.

[10] G. Lecerf. Computing the equidimensional decomposition of an algebraic closed set by means of lifting fibers. J. Complexity 19(4):564-596, 2003.

[11] A. Leykin and J. Verschelde. PHCmaple: A Maple Interface to the Numerical Homotopy Algorithms in PHCpack. In Quoc-Nam Tran, ed., Proceedings of the Tenth International Conference on Applications of Computer Algebra (ACA'04), pages 139-147, 2004.

[12] E. Mansfield. Differential Gröbner Bases. Ph.D. thesis, Univ. of Sydney, 1991.

[13] J.F. Pommaret. Systems of Partial Differential Equations and Lie Pseudogroups. Gordon and Breach Science Publishers, Inc. 1978.

[14] G. Reid, C. Smith, and J. Verschelde. Geometric completion of differential systems using numeric-symbolic continuation. SIGSAM Bulletin 36(2):1-17, 2002.

[15] G.J. Reid, D.T. Weih and A.D. Wittkopf. A Point symmetry group of a differential equation which cannot be found using infinitesimal methods. In Modern Group Analysis: Advanced Analytical and Computational Methods in Mathematical Physics. Edited by N.H. Ibragimov, M. Torrisi and A. Valenti. Kluwer, Dordrecht, 93-99, 1993.

[16] G.J. Reid, A.D. Wittkopf and A. Boulton. Reduction of systems of nonlinear partial differential equations to simplified involutive forms. Eur. J. of Appl. Math. 7: 604-635.

[17] G.J. Reid, P. Lin, and A.D. Wittkopf. Differential elimination-completion algorithms for DAE and PDAE. Studies in Applied Math. 106(1): 1-45, 2001.

[18] C.J. Rust, Rankings of derivatives for elimination algorithms and formal solvability of analytic partial differential equations, Ph.D. Thesis, University of Chicago, 1998.

[19] A. Sedoglavic. A probabilistic algorithm to test local algebraic observability in polynomial time. $J$. Symbolic Computation 33(5): 735-755, 2002.

[20] W.M. Seiler. Involution - The formal theory of differential equations and its applications in computer algebra and numerical analysis. Habilitation Thesis, Univ. of Mannheim, 2002.

[21] A.J. Sommese and J. Verschelde. Numerical homotopies to compute generic points on positive dimensional algebraic sets. Journal of Complexity 16(3):572-602, 2000.

[22] A.J. Sommese, J. Verschelde, and C.W. Wampler. Numerical irreducible decomposition using PHCpack. In M. Joswig and N. Takayama, editors, Algebra, Geometry, and Software Systems, pages 109-130. Springer-Verlag, 2003.

[23] A.J. Sommese, J. Verschelde, and C.W. Wampler. Homotopies for intersecting solution components of polynomial systems. SIAM J. Numer. Anal. 42(4):1552-1571, 2004.

[24] A.J. Sommese and C.W. Wampler. Numerical algebraic geometry. In The Mathematics of Numerical Analysis, Volume 32 of Lectures in Applied Mathematics, edited by J. Renegar, M. Shub, and S. Smale, 749-763, 1996. Proceedings of the AMS-SIAM Summer Seminar in Applied Mathematics, Park City, Utah, July 17-August 11, 1995, Park City, Utah.

[25] A.J. Sommese and C.W. Wampler. The Numerical solution of systems of polynomials arising in engineering and science. World Scientific Press, Singapore, 2005.

[26] J. Tuomela and T. Arponen. On the numerical solution of involutive ordinary differential systems. IMA J. Numer. Anal. 20: 561-599, 2000.

[27] J. Verschelde. Algorithm 795: PHCpack: A general-purpose solver for polynomial systems by homotopy continuation. ACM Transactions on Mathematical Software 25(2): 251-276, 1999. Software available at http://www.math.uic.edu/ jan.

[28] A. Wittkopf and G.J. Reid. Fast differential elimination in C: The CDiffElim environment. Computer Physics Communications, 139: 192-217, 2001.

[29] A. Wittkopf. Algorithms and Implementations for Differential Elimination. Ph.D. Thesis, Simon Fraser University, 2004.

[30] W.-T. Wu. On the foundations of algebraic differential geometry. Mathematics-Mechanization Research Preprint No. 3, pages 1-26, 1989. 